\title{
Artilleurs canadiens-français dans la libération du nord de la France, de la Belgique et de la Hollande (septembre-novembre 1944) (suite)
}

\author{
Jacques Gouin
}

Volume 17, numéro 3, décembre 1963

URI : https://id.erudit.org/iderudit/302287ar

DOI : https://doi.org/10.7202/302287ar

Aller au sommaire du numéro

Éditeur(s)

Institut d'histoire de l'Amérique française

ISSN

0035-2357 (imprimé)

1492-1383 (numérique)

Découvrir la revue

\section{Citer cet article}

Gouin, J. (1963). Artilleurs canadiens-français dans la libération du nord de la France, de la Belgique et de la Hollande (septembre-novembre 1944) (suite). Revue d'histoire de l'Amérique française, 17(3), 325-339.

https://doi.org/10.7202/302287ar d'utilisation que vous pouvez consulter en ligne. 


\section{ARTILLEURS CANADIENS-FRANÇAIS DANS LA LIBÉRATION DU NORD DE LA FRANCE, DE LA BELGIQUE ET DE LA HOLLANDE* (septembre-novembre 1944) (suite)}

6e article

\section{DE MALDEGEM Ã NIMEGUE - BATAILLE DE L'ESCAUT}

\section{DEFENSES ALLEMANDES}

Comme nous l'avons déjà vu, la nécessité d'ouvrir l'accès au port d'Anvers s'était révélée dès la première semaine de septembre, alors que le nettoyage des ports de la Manche était encore en cours. Or, pendant que la $1^{\text {re }}$ armée canadienne était tout entière occupée à libérer ces ports, le général von Zangen, commandant la $15^{\mathrm{e}}$ armée allemande, en profitait pour renforcer le canal Léopold, en prévision précisément d'une tentative des Alliés visant à ouvrir l'estuaire de l'Escaut et ainsi donner accès à Anvers ${ }^{1}$. De fait, dès le 6 septembre, von Zangen avait établi une ligne défensive puissante le long du canal Léopold, couverte par les batteries côtières de Cadzand et de Breskens ${ }^{2}$.

Afin de donner une idée de ce qui attendait le Régiment dans cette nouvelle bataille, rappelons que la $64^{\mathrm{e}}$ division allemande, chargée de défendre les approches de Breskens, était formée de combattants qui avaient connu les pires combats de Russie et qui avaient juré de combattre jusqu'au dernier homme ${ }^{3}$. Sans compter les difficultés terribles qui attendaient l'infanterie", 244-257.

*Voir notre Revue, XVI: 240-253, 353-368, 536-548; XVII: 70-86,

${ }_{1}$ R. W. Thompson, The Eighty-Five Days: The Story of the Battle of the Scheldt, Ballantine Books, (New York, 1957), 31 et 75.

2 Ibid., 59.

3 Ibid., 79 et 106.

4 Ibid., 77. 
il convient de dire que l'artillerie ne se trouvait guère en meilleure posture, puisque le terrain était presque entièrement inondé et n'offrait aucun endroit pouvant servir de poste d'observation ${ }^{5}$.

Le $1^{\text {er }}$ octobre, date de la reddition de Calais, le Régiment était en route pour entreprendre sa nouvelle campagne. Après un bref arrêt à Dixmude, il était en batterie autour de Maldegem, en Belgique, le 4 octobre, où il devait demeurer jusqu'au $19^{6}$. Dès le lendemain de l'arrivée du Régiment à Maldegem, le commandant et l'adjudant se rendaient au QG du $2^{\mathrm{e}}$ groupe, à 5 heures de l'après-midi, pour y chercher les ordres nécessaires à l'attaque imminente qui se préparait sur le canal Léopold. De retour au Régiment, le commandant déléguait le major Lahaie, le capitaine Dupuis et le capitaine Chabot au QG de la $7^{\mathrm{e}}$ brigade d'infanterie canadienne, où ces trois officiers devaient assurer l'appui du Régiment lors de l'attaque prévue pour le lendemain, et au cours de laquelle nos troupes devaient forcer le passage du canal Léopold ?.

\section{LE RÉGIMENT PARALYSE LES CONTRE-ATTAQUES ENNEMIES}

L'heure $\mathrm{H}$ étant fixée à 5.30 du matin du 6 octobre, le Régiment ouvrait le feu dès $3 \mathrm{~h}$. du matin. A l'aube, le tir se mit à ralentir, pour reprendre dans l'après-midi et la soirée. “... L'artillerie gronde partout, préludant à une autre déroute pour l'ennemi", écrivait ce jour-là un officier du Régiment ${ }^{8}$. En fait, dès ce premier jour de l'attaque, la $7^{\mathrm{e}}$ brigade, soutenue par le feu du Régiment, parvenait à faire traverser deux de ses bataillons au-delà du canal, ainsi que deux compagnies de son troisième bataillon. D'autre part, la $8^{\circ}$ brigade ne bougeait pas et aucune nouvelle ne parvenait de la $9^{\mathrm{e}}$ brigade, qui devait débarquer sur la plage au nord de l'ennemi ${ }^{9}$.

Dans la nuit du 7 octobre, de nombreuses contre-attaques allemandes nécessitèrent un violent tir d'artillerie qui les repoussa

$5 \mathrm{Ib} i d ., 78$.

6 Lieut.-col. Gagnon, etc., The History of the 4 th Canadian Medium Regt $R C A, 11$.

7 Journal de guerre du Régiment, XXXI: 2.

8 Archives de l'auteur [Maldegem], 6 oct. 1944.

9 Journal de guerre du Régiment, XXXI: 2. 
toutes avec de lourdes pertes. L'opération (du nom de "Switchback"), - dirigée par le lieutenant-général Simmonds, en l'absence du général Crerar hospitalisé en Angleterre, - qu'on avait cru devoir prendre quelques jours seulement, allait se prolonger pendant des semaines ${ }^{10}$. Ce même 7 octobre, von Zangen avait émis l'ordre du jour suivant à son armée :

La défense des approches du port d'Anvers représente une tâche qui est décisive pour la conduite future de la guerre... Par conséquent, je donne ordre aux officiers commandants, aussi bien qu'aux officiers chargés de répandre la doctrine nationalesocialiste, d'apprendre aux troupes, de la façon la plus claire et la plus précise, le fait suivant: Anvers est, après Hambourg, le plus grand port d'Europe... ${ }^{11}$.

Dans ce même ordre du jour, von Zangen ajoutait:

Quand ils se seront emparés des fortifications de l'Escaut, les Britanniques [et les Canadiens] se trouveront enfin en mesure de débarquer d'énormes masses de matériel dans un grand port parfaitement protégé. Avec ce matériel, ils pourraient porter un coup mortel aux plaines de l'Allemagne du Nord et à Berlin, avant l'arrivée de l'hiver ${ }^{12}$.

On voit donc toute l'importance que les Allemands attachaient à une résistance opiniâtre tout le long de l'estuaire de l'Escaut. Aussi, n'est-il pas étonnant de voir cette campagne se prolonger si longtemps.

\section{LE RÉGIMENT SE RÉSIGNE À UNE CAMPAGNE D'HIVER}

Le 8 octobre, le "padre" disait la messe au QG régimentaire, alors que tous se résignaient à entreprendre une campagne d'hiver, espérant toujours toutefois un effondrement soudain de l'Allemagne. En guise de consolation, le Régiment recevait le message suivant du QG du $2^{\mathrm{e}}$ groupe dans l'après-midi: "Infan-

10 Ibid.

11 Major M. Shulman, La Défaite allemande à l'Ouest, traduit de l'anglais par le capitaine de corvette André Cogniet (Paris, Payot, 1948), 238.

12 Ibid., 239. 
terie très satisfaite de votre feu harcelant. Vous avez fait taire les Boches. L'infanterie peut maintenant se reposer un peu ${ }^{13}$." Le Régiment pouvait donc se dire qu'il servait à quelque chose, et très utilement.

Le 9 octobre, il y avait très peu de changement dans la situation tactique en face du Régiment; mais, plus au nord, la $9^{\text {e }}$ brigade réussissait à débarquer sur la rive sud de l'estuaire de l'Escaut, à environ 7 milles au nord-est de la position occupée par le Régiment. Les Allemands se trouvaient donc de nouveau pris dans une trappe, comme à Falaise.

\section{LE RÉGIMENT EST CHOISI POUR UNE INSPECTION PAR LE MINISTRE DE LA DÉFENSE NATIONALE}

Ce jour-là, un nouvel honneur allait échoir au Régiment. En effet, il était choisi parmi tous les régiments d'artillerie du $2^{\text {e }}$ groupe pour faire l'objet d'une inspection officielle par le ministre de la Défense nationale, le col. Ralston. D'autre part, disons tout de suite que, le lendemain, après avoir attendu pendant des heures sous une pluie torrentielle la visite du distingué visiteur, ce dernier ne se montrait pas. Du moins, l'honneur était sauf, même s'il dut inspirer force jurons aux pauvres canonniers trempés jusqu'aux os, qui eussent sans doute préféré tirer du canon que de se faire examiner de la tête au pied par un ministre ${ }^{14}$. En fait, le Régiment avait été choisi par le brigadier Suttie, commandant du $2^{\mathrm{e}}$ groupe, pour fournir la garde d'honneur, car, avait-il dit, "this unit is the one that can put up the best show ${ }^{15 "}$.

\section{LE REGIMENT REPOUSSE DE NOUVELLES CONTRE-ATTAQUES ENNEMIES}

Peu après le crépuscule, le 10 octobre, l'ennemi déclenchait plusieurs attaques contre la tête de pont établie par la $7^{\mathrm{e}}$ brigade au nord du canal Léopold, mais toutes étaient repoussées "avec

13 Journal de guerre du Régiment, XXXI : 2-3 (trad.)

14 Ibid.

15 Ibid. 
de lourdes pertes... attribuables à notre feu", rapporte le Journal de guerre du Régiment ${ }^{16}$.

Le lendemain, le canon $\mathrm{A}$ de la $50^{\mathrm{e}}$ batterie tirait à lui seul 62 obus sur une batterie ennemie, dont 14 frappèrent la cible directement, grâce à l'observation du capitaine Dupuis. D'autre part, l'ennemi contre-attaquait sans cesse et le combat devenait acharné, le pire depuis le débarquement ${ }^{17}$. En fait, le Journal de guerre du Regina Rifles rapportait, pour sa part, le 12 octobre:

On est d'avis qu'il ne s'était pas livré de combats plus acharnés depuis le jour J ... Sur notre seul front, l'artillerie a lancé 2,000 obus en 90 minutes, le soir du 10 octobre... Mais nous croyons que ces efforts ont été couronnés de succès ${ }^{18}$.

Ce même 12 octobre, un officier rendait le témoignage suivant à l'efficacité de la présence du commandant au Régiment:

Tout va bien au Régiment. Notre colonel est revenu complètement rétabli de sa pleurésie et tout marche mieux, car sans lui on sentait qu'il manquait une poigne solide ${ }^{19}$.

\section{LUTTE ACHARNEE MALGRE L'EFFICACITÉ DU REGIMENT}

Le 13 octobre, alors que les premiers V-2 commençaient à tomber sur Anvers, Hitler ordonnait que l'estuaire de l'Escaut soit tenu à tout prix. Le général Eisenhower ordonnait, pour sa part, qu'il soit nettoyé à tout prix: c'était donc une lutte acharnée qui allait s'engager ${ }^{20}$. De son côté, le Régiment effectuait un tir de deux heures, juste après minuit, sans toutefois qu'aucune avance notable n'en résulte. Mais ce n'est pas dire que le tir du Régiment manquât d'efficacité. Au contraire, voici deux témoignages qui en disent long sur la précision de tir du

16 Ibid., 4.

17 Ibid.

18 Col. C. P. Stacey, Histoire officielle de la participation de l'armée canadienne à la seconde guerre mondiale, Vol. III, La Campagne de la victoire: les opérations dans le nord-ouest de l'Europe (1944-1945) (Ottawa, Imprimeur de la Reine), 418.

19 Archives de l'auteur [Maldegem], 12 oct. 1944.

20 Thompson, op. cit., 92 . 
Régiment. Le Journal de guerre de cette date renferme en effet cette inscription :

Le lieut.-col. Wyatt, ancien commandant du $7^{\mathrm{e}}$ régiment d'artillerie moyenne, aurait dit récemment à l'Unité de renfort de l'armée canadienne, en Angleterre, dans une causerie prononcée devant 150 officiers, "that the 4 Med was the best Canadian Medium Regt" ${ }^{21}$.

Et le lendemain 14 octobre, une lettre d'un officier du Régiment renfermait le passage suivant:

... Après Falaise, le général polonais [de qui relevait alors le Régiment] donna le crédit de la victoire comme suit: "Mon régiment de reconnaissance, et deuxièmement, mon régiment d'artillerie moyenne !" Nous étions fous de joie... Après Falaise, nous avons laissé les Polonais pour quelque temps. Or, ceux-ci, se préparant à partir pour la Belgique, demandèrent un régiment d'artillerie moyenne. Les autorités leur ont offert un autre régiment, de façon à ce que nous puissions nous reposer un peu. Mais aussitôt, la réponse arriva: "C'est le $4 \mathrm{e}$ que nous voulons et pas d'autre." C'est ainsi que nous sommes partis sans avoir eu beaucoup de temps pour nous reposer. Les artilleurs grognaient un peu, mais au fond ils étaient contents, car ils savaient que nous allions tirer des obus "en masse" ${ }^{22}$.

\section{LES CANADIENS VERS BRESKENS}

Le 14 octobre, alors que le Régiment entreprenait son onzième jour à Maldegem, qui commençait à rappeler un peu Vaucelles, moins les bombardements d'aviation et d'artillerie ${ }^{23}$, les trois brigades canadiennes avaient pénétré à l'intérieur de la poche de Breskens, que les mortiers ennemis ne cessaient pour autant de harceler avec une précision mortelle ${ }^{24}$. En effet, résolus plus que jamais à résister, les Allemands recevaient, ce jour même, du général Eberding, commandant la $64^{\mathrm{e}}$ division allemande devant Breskens, l'ordre suivant:

21 Journal de guerre du Régiment, XXXI: 4-5.

22 Archives de l'auteur [Maldegem], 14 oct. 1944.

23 Journal de guerre du Régiment, XXXI: 5.

24 Thompson, op. cit., 111. 
Tout homme qui se rend, quelles que soient les circonstances, sera considéré comme un déserteur. La Division doit tenir et mourir. Dans les cas où les noms des déserteurs seront connus, ils seront portés à la connaissance de la population civile en Allemagne et leurs parents seront considérés comme des ennemis du peuple allemand ${ }^{25}$.

C'est dire avec quel acharnement ces vétérans de Russie se voyaient forcés de résister aux assauts meurtriers des Canadiens.

Le 15 octobre, il y avait messe à 9 h. 30 du matin au Régiment. Dans la journée, l'adjudant se rendait à Gand pour y chercher le capitaine Poulin, enfin remis de sa blessure subie en Normandie, sur la route de Falaise. De retour au Régiment, le capitaine Poulin était nommé commandant de la troupe $B$, de la $50^{\mathrm{e}}$ batterie. Dans la même journée, le capitaine Sévigny allait remplacer le capitaine Chabot au poste d'observation, et le major Tremblay, en compagnie du "padre", se rendait à Bruges pour préparer des visites éducatives à l'intention des hommes du Régiment ${ }^{26}$.

Le 16 octobre, Eede tombait entre les mains de la $7^{\text {e }}$ brigade, qui poursuivait ensuite son avance vers Middlebourg. A la droite de la $7^{\mathrm{e}}$ bridage, les $8^{\mathrm{e}}$ et $10^{\mathrm{e}}$ brigades avançaient constamment vers l'ouest et consolidaient leur tête de pont. Ce même jour, le commandant allait préparer un nouveau poste d'observation que devait occuper le lieutenant Caron le lendemain ${ }^{27}$. Le 18 , le capitaine Poulin et le lieutenant Bourbonnière accompagnaient le commandant et le major Archer au poste d'observation et y effectuaient quelques tirs pendant la journée, malgré un feu de mortiers ennemis qui réussit à détruire une jeep de la $50^{\mathrm{e}}$ batterie, sans heureusement blesser personne ${ }^{28}$.

Le 19 octobre, il faisait un temps affreux. A l'aube, la $157^{\mathrm{e}}$ brigade britannique venait remplacer la $7^{\mathrm{e}}$ brigade canadienne, qui s'en allait en réserve de la $9^{\mathrm{e}}$ brigade canadienne.

25 Ibid., 91 (trad.) ; cité aussi dans Wilmot, op. cit., et dans le Journal de guerre de la $7^{\mathrm{e}}$ brigade d'infanterie canadienne.

26 Journal de guerre du Régiment, XXXI: 5.

27 Ibid.

28 Ibid., 6. 
On enregistrait de bonnes avances ce jour-là. En effet, à midi, la moitié de la poche de Breskens était libérée, sans tir ou presque de la part du Régiment. L'ennemi se trouvait enfin forcé de se replier ${ }^{29}$. Le lendemain, le Régiment recevait la bonne nouvelle que des congés de 48 heures allaient bientôt être accordés pour Bruxelles ou Anvers ${ }^{30}$. Ce même jour, 20 octobre, le Journal de guerre du Régiment notait que la résistance ennemie faiblissait tout le long du front de Breskens, à tel point qu'à 4 heures de l'après-midi, la $50^{\mathrm{e}}$ batterie avançait vers une nouvelle position, à quelques verges du canal Léopold, et que le lendemain le reste du Régiment avançait à son tour ${ }^{31}$.

Le 21 octobre, le QG du Régiment était établi dans le petit village de Scheewage, récemment évacué par les civils. Le même jour, le capitaine Moss était délégué par le commandant auprès de la $4^{\mathrm{e}}$ brigade du service spécial, en vue d'une attaque imminente contre Flessingue ${ }^{32}$. Le lendemain, après que le Régiment eut effectué un violent tir de harcèlement sur l'ennemi, Breskens tombait aux mains des troupes canadiennes.

\section{LE REGIMENT SE CLASSE PREMIER DANS L'ARMÉE CANADIENNE}

Pour couronner cette victoire, le Régiment apprenait le même jour qu'il s'était classé premier dans le $2^{e}$ groupe au cours de la dernière campagne d'emprunt de la Victoire, et que le $2^{\mathrm{e}}$ groupe s'était classé premier de toute l'armée canadienne. C'est donc dire que le Régiment, en plus de ses faits d'armes, se classait premier de toute l'armée canadienne pour ce qui était de l'emprunt de la Victoire ${ }^{33}$.

Un air de victoire flottait en effet dans l'atmosphère à cette date, comme en témoigne ce passage d'une lettre d'un officier du Régiment:

....Nous avons trouvé un déserteur allemand caché

dans une maison depuis plusieurs jours ... A l'aide

29 Ibid., 6-7.

30 Archives de l'auteur [Maldigem], 20 oct. 1944.

31 Journal de guerre du Régiment, XXXI: 7.

32 Ibid.

33 Ibid., 7-8. 
d'un dictionnaire, j'ai causé avec lui. D'après lui, 80 p. 100 des Allemands sont contre la guerre et en ont jusqu'au cou. Seuls les officiers et les SS s'obstinent avec l'acharnement du désespoir à retarder le désastre qui les attend. "Deutschland und Hitler kaput”, me disait-il toujours ... D'après ce prisonnier, la guerre sera finie au mois de novembre ! Evidemment, sa volonté de voir tout fini l'égare un peu. Je ne me suis fait aucune illusion sur ses prédictions ${ }^{34}$.

Un autre passage de cette même lettre donne une idée du moral du Régiment, malgré les intempéries et les difficultés de cette campagne:

... L'hiver commence à se faire sentir désagréablement, surtout dans un pays aussi humide que celui-ci. Il pleut souvent, et mon admiration est sans bornes pour nos pauvres canonniers qui pataugent dans la boue jour et nuit. Malgré tout, leur moral est excellent. Notre Régiment est déjà connu en Angleterre, depuis notre chevauchée fantastique avec la division polonaise ${ }^{35}$.

\section{LE REGIMENT ENTRE EN HOLLANDE}

Le 23 octobre, le Régiment avançait de nouveau, cette fois pour entrer en Hollande. En effet, juste avant le déjeuner, le Régiment était en batterie à Ijzendijke. Les canons se trouvaient plus que jamais enfoncés dans la boue, tous les champs étant inondés. Le Régiment se trouvait à remplacer à cet endroit le $9^{\mathbf{e}}$ régiment d'artillerie britannique, de sorte que, pendant quelque temps, il reçut des ordres de tir non seulement du $2^{\circ}$ groupe canadien mais du $9^{\mathrm{e}}$ groupe britannique. Pendant cette seule journée, le Régiment tirait environ 150 obus sur l'ennemi ${ }^{36}$.

\section{PRÉPARATIFS EN VUE DU DÉBARQUEMENT À FLESSINGUE}

Le lendemain, 24 octobre, le commandant se rendait auprès du QG de la $4^{\text {e }}$ brigade du service spécial avec les canonniers

${ }^{34}$ Archives de l'auteur [Scheewage], 22 oct. 1944.

35 Ibid.

36 Journal de guerre du Régiment, XXXI: 8. 
Femia, Godding, Leclerc, Snider, Casey et Trotter, qui devaient se préparer à travailler avec le capitaine Moss, lors du débarquement imminent à Flessingue. Cette journée-là, un malheureux accident devait causer trois morts dans le Régiment. En effet, un peu avant 2 heures de l'après-midi, le canon $\mathrm{n}^{\circ} 2$ de la troupe A de la $50^{\mathrm{e}}$ batterie éclatait, causant la mort du caporal d'artillerie L. Simard, et des canonniers P.-E. Caron et E. Ducharme. Deux autres canonniers, Généreux et Sinnett, étaient légèrement blessés ${ }^{37}$. Dès le lendemain, le "padre" enterrait les trois morts dans le cimetière d'Ijzendijke.

A cette date, les $2^{\mathrm{e}}$ et $3^{\mathrm{e}}$ divisions canadiennes avaient fermé le col de l'isthme du Beveland méridional et ouvert le port de Breskens, deux opérations préliminaires essentielles à la prise de Walcheren et au nettoyage de la rive nord de l'Escaut ${ }^{38}$. Les sept dixièmes de l'estuaire de l'Escaut se trouvaient ainsi libérés et 5,000 prisonniers allaient grossir les cages de l'armée canadienne. L'ennemi ne tenait plus que Slouis, Cadzand et Knocke ${ }^{39}$. Le lendemain, 1,000 autres prisonniers étaient capturés. Voici un extrait d'une lettre d'un officier du Régiment, en date du 26 octobre, qui donne une idée de l'aspect des prisonniers à cette époque-là :

... Nous n'avons jamais été si occupés depuis la bataille de Falaise... Nous ne comptons plus les tonnes d'obus que les Allemands encaissent chaque jour et chaque nuit, sans répit; c'est incroyable. J'ai visité hier, tout près d'ici, une cage de prisonniers; c'était pitoyable: des jeunes et des presque vieillards à barbe longue, en haillons, - c'est l'image actuelle de la fameuse Werhmacht. Si quelques officiers fanatiques cessaient de poursuivre une lutte inégale et meurtrière, la guerre finirait ce soir. C'est l'entêtement du désespoir qui prolonge inutilement ce conflit horrible ${ }^{40}$.

Et voici, dans cette même lettre, une impression qui résume assez bien le sentiment qu'on éprouvait alors à l'égard de la

37 Ibid.

38 Thompson, op. cit., 118.

39 Journal de guerre du Régiment, XXXI: 9.

40 Archives de l'auteur [Ijzendijke], 26 oct. 1944. 
Hollande: “. . . je suis en Hollande. Mon impression n'est guère enthousiaste: de la boue, encore de la boue, toujours de la boue ${ }^{41}$."

\section{LE RÉGIMENT APPUIE LE DÉBARQUEMENT}

Le 27 octobre, les troupes canadiennes débarquaient au sud de l'isthme de Beveland. Dès le lendemain, le groupe de reconnaissance du Régiment se rendait au sud de Breskens pour préparer une nouvelle position d'où les canons allaient appuyer le débarquement sur Flessingue. Le capitaine Moss, désigné pour accompagner les commandos de la $4^{\mathrm{e}}$ brigade du service spécial dans cette opération, passait la journée au Régiment. Le lendemain, le commandant se rendait au $Q G$ du $2^{\text {e }}$ groupe pour assister à une conférence qui durait deux heures.

\section{LE RÉGIMENT EST FÉLICITÉ}

Pendant cette journée, le Régiment ne devait pas tirer beaucoup, vu que la résistance cédait partout. Mais, tard dans la soirée, il recevait l'ordre d'avancer, ce qui représentait une opération extrêmement ardue, que le Régiment effectua tout de même en un temps record, comme en fait foi la lettre suivante du brigadier Suttie, commandant du $2^{\ominus}$ groupe, adressée au commandant du Régiment dès le lendemain:

Tard hier soir, on nous a demandé d'accomplir l'impossible. Le jeu semblait en valoir la chandelle et le contrat fut accepté.

Malheureusement, le plan prévu ne fut pas exécuté et l'effort déployé pour atteindre le but peut sembler inutile.

Il est, à vrai dire, agréable de savoir que votre unité ait pu dépasser le but malgré les difficultés et les obstacles qui entravaient l'opération.

Veuillez, je vous prie, transmettre à tous vos hommes l'expression de ma gratitude pour les efforts qu'ils ont faits. Encore une fois, vous avez confirmé qu'aucune tâche n'est trop difficile pour que vous ne puissiez la mener à bonne fin ${ }^{42}$.

41 Ibid.

42 Documents personnels du brigadier J.-H.-R. Gagnon, O.B.E., 30 oct. 1944 (Traduction). 
Le même jour, le commandant du Régiment répondait ce qui suit:

... Tous ceux qui sont sous mon commandement comprennent la situation et se sentent plutôt fiers d'effectuer des déplacements rapides et difficiles, comme s'il s'agissait d'une routine. Je dois ajouter que l'avance de ce matin représentait le meilleur entraînement qu'on puisse se procurer dans les conditions actuelles.

De toute façon, l'effort n'a pas été perdu. Toutes les munitions au sol auront été tirées sur l'ennemi avant demain matin ...43.

\section{LE SORT DES CIVILS HOLLANDAIS}

Pour peu qu'on puisse s'imaginer l'état du terrain dans cette région de la Hollande, à ce moment-là, on saisira toute la signification de l'échange de lettres ci-dessus en disant que le Régiment, parti d'Ijzendijke à 8 heures du matin, était déjà en batterie autour de Breskens à 10 heures, et prêt à tirer à 10 h. 30 ! Pareil déplacement ne se faisait pas, cependant, sans secouer plutôt brutalement les pauvres Hollandais, comme en fait foi l'extrait de lettre suivant d'un officier du Régiment:

... La misère en Hollande n'a d'égale que celle que j'ai vue en Normandie, lors des premiers combats farouches autour de Caen. Pendant deux jours et deux nuits, j'ai vu défiler des réfugiés de toutes les conditions, dans la boue et sous une pluie torrentielle. Des femmes portaient des bébés dans leurs bras. Les hommes, chaussés de gros sabots, tiraient des charrettes improvisées, et souvent toute une famille devait s'arrêter sur le bord de la route pour ne pas bloquer les convois militaires. La guerre, c'est terrible ... De voir tant d'innocents souffrir ainsi, c'est quasi incompréhensible ...44.

Le 31 octobre, le Régiment tirait 1600 obus. Les prisonniers se rendaient cette fois en disant: "Votre artillerie est trop terrible pour nous ${ }^{45 "}$. A midi, le Régiment recevait de nouveau

43 Ibid.

44 Archives de l'auteur [près de Breskens], 30 oct. 1944.

45 Journal de guerre du Régiment, XXXI: 11. 
l'ordre d'avancer. Cette fois, l'opération "Switchback" (liquidation de la poche de Breskens) était terminée, et une nouvelle opération ("Infatuate") allait être entreprise pour capturer l'île de Walcheren et les îles Beveland, où se trouvaient encore 11,000 Allemands. Tout était prêt pour recevoir des ordres de feu du capitaine Moss, attaché auprès de la $4^{\mathrm{e}}$ brigade du service spécial ${ }^{46}$.

\section{PUISSANCE DE L'ARTILLERIE ANGLO-CANADIENNE}

Pour la seule attaque sur Flessingue, le brigadier A. Bruce Matthews, qui était chargé de coordonner le tir de l'artillerie, disposait de 314 canons. "L'efficacité de ses pièces [fut] formidable dans la région de Flessingue", écrit le col. Stacey ${ }^{47}$. D'autre part, voici ce qu'écrit $\mathrm{R}$. W. Thompson, dans son livre sur la bataille de l'Escaut, de la puissance de l'artillerie canadienne et britannique au moment de la prise de Flessingue:

A tremendous artillery barrage made the night hideous, and revealed the facade of Flushing in dark silhouette, its great gantries standing out like the stricken trees of a petrified forest... The traffic in shells wiffled and woofed overhead in strange sibilant layers of overtones and undertones... the guns beating out an ominous rythm through the heavy cloud banks ${ }^{48}$.

\section{ACHARNEMENT DE L'ENNEMI}

Malgré cela, l'ennemi ne semblait pas vouloir encore lâcher prise, car, en plus de ce puissant barrage, il fallut par la suite répondre à des appels urgents et chroniques pour appuyer les troupes déjà engagées dans Flessingue même. De fait, les défenses de l'île de Walcheren, mises au point avec toute l'habileté des Allemands pour ce genre de chose, étaient sans doute les plus puissantes au monde. Toutes les digues étaient hérissées de canons, le plus souvent protégés par une quinzaine de pieds d'épaisseur de béton ${ }^{49}$.

\section{Ibid.}

47 Stacey, op. cit., 431.

48 Thompson, op. cit., 148-149-150.

49 Ibid., 143. 
Pourtant, malgré ces défenses formidables, dès le $1^{\text {er }}$ novembre, avant la tombée de la nuit, Flessingue cédait aux troupes canadiennes. Le 2 , les canons du Régiment répondaient encore sans cesse aux appels du capitaine Moss et enregistraient de nombreux coups directs, bien que les canonniers dussent patauger dans la boue jusqu'aux genoux ${ }^{50}$.

Le 5 novembre, après la prise de Flessingue et la capture de 5,000 autres prisonniers par les troupes canadiennes, le Régiment apprenait qu'il allait enfin prendre quelques jours de repos bien mérité dans la région de St-Nicolas, en Belgique. Le lendemain, le commandant se rendait dans cette localité pour préparer l'arrivée du Régiment. Celui-ci partait à $8 \mathrm{~h}$. $30 \mathrm{du}$ matin et arrivait à destination à 8 heures du soir, en pleine obscurité ${ }^{51}$.

\section{REPOS VITE INTERROMPU}

Le 8 novembre, il y avait danse pour les officiers à St-Nicolas mais, malheureusement, peu avant minuit, le Régiment recevait l'ordre de se préparer à partir dès le lendemain matin. Cette fois, il s'agissait pour lui de se rendre dans le saillant de Nimègue, à l'extrémité du front occupé par l'armée canadienne. Tôt dans la matinée du 9, le groupe de reconnaissance partait, et était suivi à $10 \mathrm{~h}$. 30 par le gros du Régiment. Celui-ci allait franchir près de 100 milles dans la journée, sous la pluie et la grêle, et dans la boue, après avoir contourné Anvers et passé par Tilburg et s'Hertogenbosch. Après une halte à Nistelrode pour la nuit, le Régiment reprenait la route le lendemain pour se rendre jusque dans le saillant de Nimègue où, pour la première fois, il se trouvait en compagnie de troupes américaines, celles de la $101^{\mathrm{e}}$ division de parachutistes. Le Régiment arrivait à destination à $3 \mathrm{~h}$. 30 , pour s'établir près de Lœenen, au nord de la Waal, à quelques milles à l'est de Nimègue et au sud d'Arnhem. Le Régiment devait rester dans ce secteur jusqu'au 8 février 1945, date de la dernière grande offensive contre le nord de l'Allemagne ${ }^{52}$.

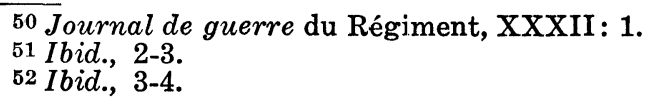


FIN DE LA BATAILLE DE L'ESCAUT

Une autre phase était terminée pour le Régiment. Il venait de participer avec éclat à cette dure bataille que fut la libération de l'estuaire de l'Escaut. Comme l'écrit le col. Stacey:

La lutte avait été dure et sanglante... La bataille se livrait sur un terrain extrêmement défavorable et contre un ennemi déterminé qui comprenait pleinement l'importance du terrain qu'il défendait ${ }^{53}$.

Grâce à ce magnifique effort de l'armée canadienne, et en partie grâce aussi sans doute à la rapidité et à la précision du tir du Régiment, le port d'Anvers était ouvert aux Alliés le 26 novembre 1944. Le $1^{\text {er }}$ décembre, les premières 10,000 tonnes d'approvisionnements étaient déchargées dans ce port, dont on avait attendu l'ouverture avec tant d'impatience ${ }^{54}$. Le Régiment pouvait se dire qu'il avait encore une fois participé à une série d'opérations indispensables à la victoire finale.

(à suivre)

JACQUES GOUIN,
ex-lieutenant d'artillerie,
diplômé en sciences politiques (Ottawa),
correspondant canadien à la Revue d'Histoire
de la 2e guerre mondiale (France)
chef adjoint du Bureau des traductions et
rédacteur de l'édition française du Journal
de l'Armée canadienne, ministère de
la Défense nationale, Ottawa.

53 Stacey, op. cit., 450.

54 Thompson, 216. 